\title{
Impact of Plasmodium falciparum malaria and intermittent preventive treatment of malaria in pregnancy on the risk of malaria in infants: a systematic review
}

\author{
Abel Kakuru ${ }^{1,2^{*}} \mathbb{D}$, Sarah G. Staedke ${ }^{2}$, Grant Dorsey ${ }^{3}$, Stephen Rogerson ${ }^{4}$ and Daniel Chandramohan ${ }^{2}$
}

\begin{abstract}
Background: Studies of the association between malaria in pregnancy (MiP) and malaria during infancy have provided mixed results. A systematic review was conducted to evaluate available evidence on the impact of Plasmodium falciparum malaria infection during pregnancy, and intermittent preventive treatment of malaria during pregnancy (IPTp), on the risk of clinical malaria or parasitaemia during infancy.

Methods: MEDLINE, EMBASE, Global Health, and Malaria in Pregnancy Library databases were searched from inception to 22 May 2018 for articles published in English that reported on associations between MiP and malaria risk in infancy. Search terms included malaria, Plasmodium falciparum, pregnancy, placenta, maternal, prenatal, foetal, newborn, infant, child or offspring or preschool. Randomized controlled trials and prospective cohort studies, which followed infants for at least 6 months, were included if any of the following outcomes were reported: incidence of clinical malaria, prevalence of parasitaemia, and time to first episode of parasitaemia or clinical malaria. Substantial heterogeneity between studies precluded meta-analysis. Thus, a narrative synthesis of included studies was conducted.
\end{abstract}

Results: The search yielded 14 published studies, 10 prospective cohort studies and four randomized trials; all were conducted in sub-Saharan Africa. Infants born to mothers with parasitaemia during pregnancy were at higher risk of malaria in three of four studies that assessed this association. Placental malaria detected by microscopy or histology was associated with a higher risk of malaria during infancy in nine of 12 studies, but only one study adjusted for malaria transmission intensity. No statistically significant associations between the use of IPTp or different IPTp regimens and the risk of malaria during infancy were identified.

Conclusion: Evidence of an association between MiP and IPTp and risk of malaria in infancy is limited and of variable quality. Most studies did not adequately adjust for malaria transmission intensity shared by mothers and their infants. Further research is needed to confirm or exclude an association between MiP and malaria in infancy. Randomized trials evaluating highly effective interventions aimed at preventing MiP, such as IPTp with dihydroartemisinin-piperaquine, may help to establish a causal association between MiP and malaria in infancy.

Keywords: Malaria, Pregnancy, Infants, Intermittent preventive treatment

\footnotetext{
*Correspondence: abelkakuru@gmail.com

${ }^{2}$ London School of Hygiene and Tropical Medicine, Keppel Street, London WC1E 7HT, UK

Full list of author information is available at the end of the article
} 


\section{Background}

In sub-Saharan Africa, an estimated 30 million pregnant women are at risk of Plasmodium falciparum infection every year [1]. In areas of moderate to high malaria transmission intensity, like most parts of sub-Saharan Africa, $P$. falciparum infection in pregnant women is usually asymptomatic because adults are usually partially immune to malaria infection. However, P. falciparum infection during pregnancy can lead to placental malaria (PM). At delivery, $25 \%$ of pregnant women in sub-Saharan Africa were estimated to have PM detected by microscopy in 2007 [2]. Infection with $P$. falciparum during pregnancy has been associated with maternal morbidity such as maternal anaemia [3] and adverse birth outcomes including abortions, stillbirths, preterm delivery, and low birth weight [4-7].

The effects of $P$. falciparum infection during pregnancy on the infant may extend beyond the neonatal period [8]. Studies have shown that in utero fetal exposure to malaria antigens may negatively affect development of immunity to infectious diseases including malaria in the newborn [9-11]. Fetal exposure to P. falciparum antigens has been shown to induce malaria specific immune responses that are biased towards tolerance to malaria antigens [1214] while treatment of malaria in pregnancy (MiP) was shown to be associated with pro-inflammatory responses toward malaria specific antigens [15], suggesting that infants exposed to malaria in utero may have a higher risk of malaria during early childhood and treatment of MiP may improve anti-malarial immunity in infants. However, studies evaluating the association between MiP and malaria in infancy have shown mixed results. Some studies have reported an increased risk of clinical malaria or parasitaemia in infants born to mothers with placental malaria (PM) [16-18], while others have reported no difference in the risk of malaria in infants born to mothers with and without PM at delivery $[19,20]$. One study has suggested that infants born to primigravid mothers with PM have a lower risk of malaria [21].

Intermittent preventive treatment of MiP (IPTp) with sulfadoxine-pyrimethamine (SP), remains one of the main interventions recommended by the World Health Organization (WHO) in areas of moderate to high malaria transmission intensity mainly to improve birth outcomes [22] despite widespread resistance of malaria parasites to antifolate drugs [23]. Although IPTp-SP still improves birth outcomes in settings with antifolate resistance [24], its impact on PM and maternal parasitaemia remains minimal $[25,26]$. This continues to expose the fetus to malaria antigens which may negatively affect the health of the infant even after delivery [27]. Intermittent preventive treatment has been shown to be associated with improved infant outcomes beyond delivery such as perinatal mortality [28] but the impact of IPTp on the risk of malaria during infancy is not well known. With available promising alternative drugs for IPTp such as dihydroartemisinin piperaquine (DP) which markedly reduce both the risk of malaria parasitaemia and incidence of clinical malaria during pregnancy and reduce the prevalence of PM at delivery but does not clearly improve birth outcomes compared to IPTp-SP [25, 26, 29], possible additional benefits of IPTp to the newborn including reducing the risk of malaria in infancy may have IPTp policy implications. Understanding the impact of IPTp on the risk of malaria in infants is important in order to maximize the benefits of malaria prevention in pregnancy.

To better understand the effect of maternal parasitaemia, PM, and IPTp on the risk of malaria in infants, which may have potential to guide policy on the choice of alternative drugs for IPTp, a systematic review was conducted to examine and summarize published studies evaluating the impact of $P$. falciparum parasitaemia in pregnancy and PM, and the effect of IPTp, on the risk of clinical malaria or parasitaemia in infants.

\section{Methods}

This systematic review was conducted following the Preferred Reporting Items for Systematic Reviews and Meta-Analysis (PRISMA) guidelines [30]. The protocol for this systematic review was developed and registered with PROSPERO register (Registration number: CRD42018088869) prior to conducting the review.

\section{Selection criteria}

Original research studies were included if they were published in English and evaluated associations between $P$. falciparum infection during pregnancy or IPTp and the risk of parasitaemia or incidence of malaria in infants born to HIV-uninfected pregnant women. Only randomized controlled trials (RCTs) and prospective cohort studies in which infants were followed up for at least 6 months were included. Studies involving only HIVexposed infants, animal studies, and studies of non-falciparum malaria were excluded.

\section{Information sources and search strategy}

MEDLINE, EMBASE, Global Health, and Malaria in Pregnancy (MiP) Library [31] databases were searched from inception to 22 May 2018. All review authors participated in developing the search strategy and AK conducted the search. MEDLINE, EMBASE and Global Health databases were searched via the Ovid interface using Medical Subject Headings (MeSH) of key search terms. The MiP library [31] was searched using key search terms, including malaria, Plasmodium falciparum, 
falciparum malaria, pregnancy, placenta, maternal, foetal, prenatal, utero, new-born, infant, child, offspring, and preschool. The search was limited to journal articles reporting human studies and published in the English language. Additional studies were identified by scrutinising reference lists of studies that met the inclusion criteria and relevant review articles. A bibliography of included studies was shared with other experts in the field of MiP to assess whether all the relevant articles had been retrieved.

\section{Study selection}

Lists of titles and abstracts of retrieved articles were exported to Endnote and duplicates were removed. Retrieved titles and abstracts were assessed for eligibility at the level of titles and abstracts by the corresponding author. Full articles and abstracts of potentially relevant articles and those where there was uncertainty about whether to include or exclude the article were retrieved and assessed for eligibility by two independent reviewers (AK and DC). Where there was disagreement, it was resolved by discussing with the rest of the authors.

\section{Data extraction process}

Data were extracted by one reviewer (AK) and verified by a second reviewer (DC). A data extraction matrix in an Excel spreadsheet was developed and piloted prior to data extraction. The Excel spreadsheet included the following variables: author and year of publication, country where the study was done, study period (dates of fieldwork), transmission intensity as measured by entomological inoculation rate, study design (considered RCT if IPTp was randomized, otherwise defined as an observational study), length of follow-up, follow-up schedule, study objectives, study population, sample size, long-lasting insecticide treated net (ITN) coverage among study participants, whether IPTp was given, type and frequency of IPTp regimen, whether maternal peripheral malaria parasitaemia was measured, timing of measurement of maternal peripheral malaria parasitaemia and how it was measured, PM detection, PM case definition, when and how clinical malaria or parasitaemia were detected in the infant, study outcomes, proportion of infants born to mothers with peripheral malaria parasitaemia or PM, adjustment for potential confounding factors, losses to follow-up, proportion of infants with outcomes of interest, results including effect sizes with confidence intervals and p-values, and study strengths and limitations. Disagreements between the two reviewers were resolved by discussion. Unresolved disagreements were settled by a third reviewer (either SS or GD or SR). Corresponding authors of included studies were contacted by email for any missing or unclear information. Corresponding authors who did not respond to the first email contact were contacted two more times and if they did not respond at the third contact, they were not contacted any further.

\section{Assessment of risk of bias}

The risk of bias in individual studies was assessed by two reviewers using the Newcastle-Ottawa quality assessment scale for cohort studies [32], and the Cochrane Collaboration tool for randomized controlled trials [33]. Details of how the risk of bias was assessed in cohort studies were published in the systematic review protocol (PROSPERO Registration number: CRD42018088869). Studies were rated on the following categories: selection, comparability and outcome. For the selection category, studies were rated on the following items: representativeness of the exposed group (whether the exposed cohort was truly representative of the average from the community), selection of the non-exposed group, measurement of the exposure (if the exposed and the unexposed were from the same community), and demonstration that the outcome was not present at the start of follow-up. For the comparability category, studies were assessed based on whether the study adjusted for malaria transmission or controlled for malaria prevention during pregnancy using IPTp or ITNs. In the outcome category, studies were rated on the following items: ascertainment of outcome, whether follow-up was long enough for outcomes to occur (follow-up of at least 6 months was considered adequate), and completeness of follow-up (proportion of infants lost to follow, and whether characteristics of those lost to follow-up were reported). Each item in the selection and outcome categories was awarded a maximum of one point. The comparability category was awarded a maximum of two points. Studies were awarded a maximum of nine points. Studies that had a score of nine were rated as having medium risk of bias while those with a score of less than nine were rated as high risk.

\section{Data synthesis}

A systematic narrative synthesis of the included studies was conducted. A meta-analysis was not conducted because studies had substantial variation in the definition of malaria exposure during pregnancy, type of IPTp given, length of follow-up, and determination of primary outcome. Results were summarized using tables and in text. Tables with summary descriptions of study design, MiP exposure measurement, follow-up time, outcome measurement and results were generated. Studies were grouped in clusters according to the type of exposure (maternal peripheral malaria during pregnancy, or PM or IPTp), and outcome of interest (incidence of malaria in 
infants, prevalence of malaria parasitaemia, time to first malaria parasitaemia or first episode of malaria).

\section{Results}

\section{Study selection}

Overall, 2084 titles and abstracts were identified and retrieved from searches of electronic databases. An additional two records were identified from searching lists and contacting experts in the field (Fig. 1). Of the 2086 records, 461 duplicates were removed, and 1625 records were screened. Of these, 1582 records were excluded after review of the title and abstract, and 29 were excluded for various reasons after reviewing full text articles. Only 14 articles were deemed to be eligible for inclusion in the systematic review.

\section{Characteristics of included studies}

All 14 studies were conducted in sub-Saharan Africa (Table 1); 10 prospective cohort studies and four RCTs. Of the 10 cohort studies, IPTp-SP was given in seven studies, and IPTp was not given in two [17, 34] because they were conducted before implementation of the WHO recommendation on IPTp. Malaria transmission data in form of entomological inoculation rate (number of infectious mosquito bites per person per year) was reported in half of the included studies; 20.5 [35], 35 [18], 38 [16],

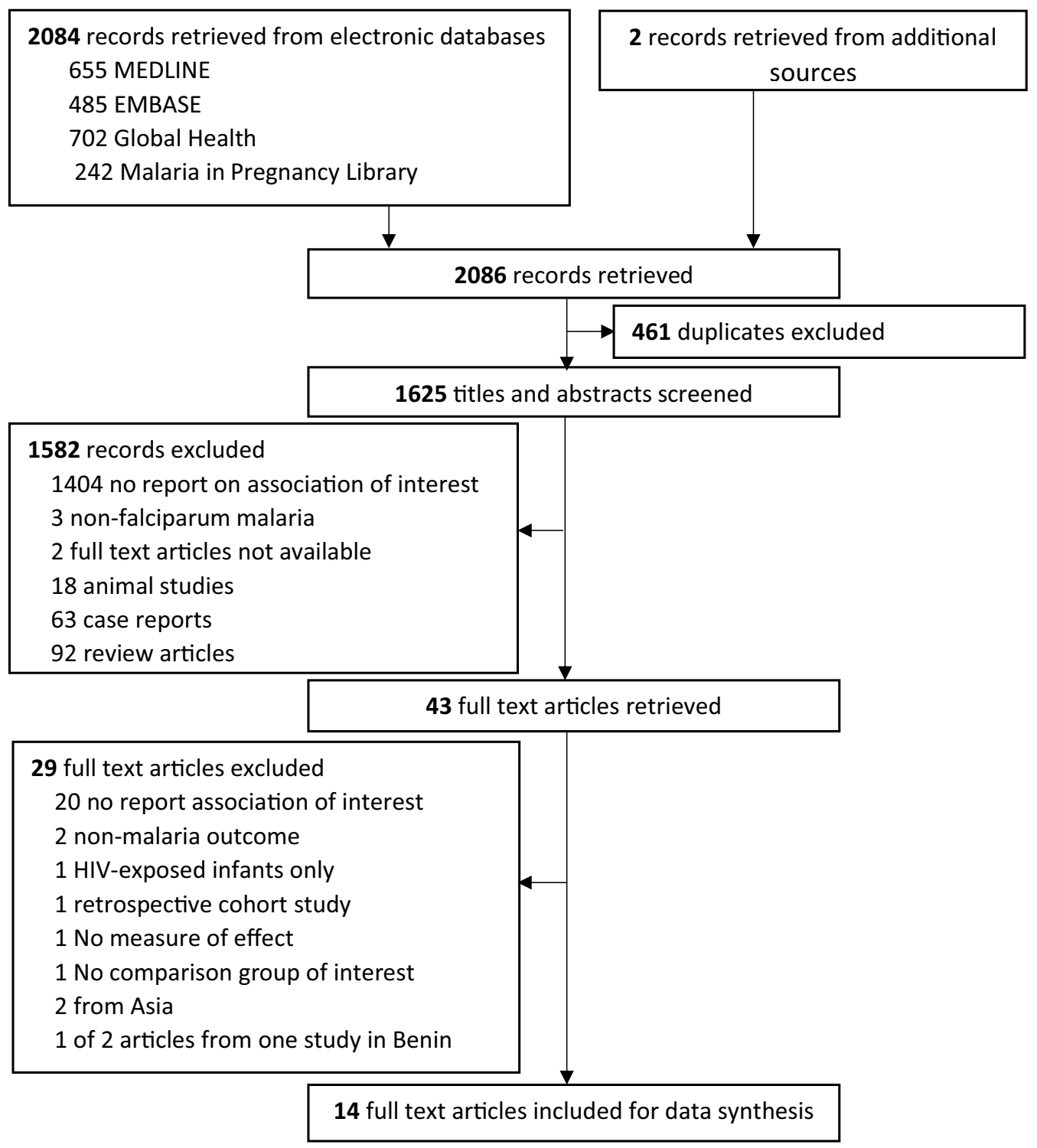

Fig. 1 Study selection results 
Table 1 Characteristics of included studies

\begin{tabular}{|c|c|c|c|c|c|c|c|c|c|}
\hline \multirow{2}{*}{$\begin{array}{l}\text { Author, year } \\
\text { of publication. } \\
\text { Country } \\
\text { (references) }\end{array}$} & \multirow[t]{2}{*}{$N$} & \multirow[t]{2}{*}{ Study design } & \multirow[t]{2}{*}{ IPTp regimens } & \multirow[t]{2}{*}{$\begin{array}{l}\text { Follow-up } \\
\text { duration }\end{array}$} & \multicolumn{3}{|c|}{$\begin{array}{l}\text { Measures of malaria } \\
\text { in pregnancy }\end{array}$} & \multicolumn{2}{|c|}{$\begin{array}{l}\text { Measures of malaria } \\
\text { during infancy }\end{array}$} \\
\hline & & & & & $\begin{array}{l}\text { Maternal } \\
\text { blood }^{\mathrm{a}}\end{array}$ & $\begin{array}{l}\text { Placental } \\
\text { blood }^{\mathbf{b}}\end{array}$ & $\begin{array}{l}\text { Placental } \\
\text { histologyc }\end{array}$ & parasitaemia & Clinical malaria \\
\hline $\begin{array}{l}\text { Tassi Yunga, } 2018 . \\
\text { Cameroon [34] }\end{array}$ & 80 & Cohort & None & 1 year & & $\checkmark$ & & $\checkmark$ & \\
\hline $\begin{array}{l}\text { Boudova, } 2017 . \\
\text { Malawi [40] }\end{array}$ & 473 & $\mathrm{RCT}$ & $\begin{array}{l}\text { SP vs CQ vs CQ } \\
\text { prophylaxis }\end{array}$ & 2 years & $\checkmark$ & $\checkmark$ & $\checkmark$ & $\checkmark$ & $\checkmark$ \\
\hline $\begin{array}{l}\text { Ruperez, } 2016 . \\
\text { Benin, Gabon, } \\
\text { Tanzania, } \\
\text { Mozambique } \\
\text { [38] }\end{array}$ & 4247 & $\mathrm{RCT}$ & SP vs MQ & 1 year & & & & & $\checkmark$ \\
\hline $\begin{array}{l}\text { Sylvester, } 2016 . \\
\text { Tanzania [41] }\end{array}$ & 206 & Cohort & Not reported & 2 years & & & $\checkmark$ & $\checkmark$ & \\
\hline $\begin{array}{l}\text { De Beaudrap, } \\
\text { 2016. Uganda } \\
\text { [37] }\end{array}$ & 832 & Cohort & SP & 1 year & $\checkmark$ & $\checkmark$ & & $\checkmark$ & \\
\hline $\begin{array}{l}\text { Awine, } 2016 . \\
\text { Ghana [19] }\end{array}$ & 988 & $\mathrm{RCT}$ & SP vs ISTp-AL & 1 year & & & $\checkmark$ & & $\checkmark$ \\
\hline $\begin{array}{l}\text { Apinjoh, } 2015 . \\
\text { Cameroon [36] }\end{array}$ & 415 & Cohort & SP & 1 year & $\checkmark$ & $\checkmark$ & $\checkmark$ & $\checkmark$ & \\
\hline $\begin{array}{l}\text { Ndibazza, } 2013 . \\
\text { Uganda [39] }\end{array}$ & 2289 & Cohort & SP & 5 years & $\checkmark$ & & & & $\checkmark$ \\
\hline $\begin{array}{l}\text { Borgella, } 2013 . \\
\text { Benin [18] }\end{array}$ & 194 & Cohort & SP & 1 year & $\checkmark$ & $\checkmark$ & & $\checkmark$ & $\checkmark$ \\
\hline $\begin{array}{l}\text { Asante, } 2013 . \\
\text { Ghana [20] }\end{array}$ & 1855 & Cohort & SP & 1 year & & & & & $\checkmark$ \\
\hline $\begin{array}{l}\text { Le Port, } 2011 . \\
\text { Benin [35] }\end{array}$ & 550 & Cohort & SP & 1 year & & $\checkmark$ & & $\checkmark$ & \\
\hline $\begin{array}{l}\text { Bardaji, } 2011 . \\
\text { Mozambique } \\
\text { [16] }\end{array}$ & 997 & $\mathrm{RCT}$ & SP vs placebo & 1 year & & $\checkmark$ & $\checkmark$ & $\checkmark$ & \\
\hline $\begin{array}{l}\text { Schwarz, } 2008 . \\
\text { Gabon [17] }\end{array}$ & 527 & Cohort & None & 2.5 years & & $\checkmark$ & & & $\checkmark$ \\
\hline $\begin{array}{l}\text { Mutabingwa, } \\
\text { 2005. Tanzania } \\
\text { [21] }\end{array}$ & 453 & Cohort & SP & 1 year & & $\checkmark$ & & $\checkmark$ & \\
\hline
\end{tabular}

$A L$ artemether lumefantrine, CQ chloroquine, IPTp intermittent preventive treatment of malaria in pregnancy, ISTp intermittent screening and treatment of malaria in pregnancy, $M Q$ mefloquine, $R C T$ randomized controlled trial, SP sulfadoxine-pyrimethamine

a Malaria detected in maternal blood by microscopy or PCR

b Placental malaria detected in placental blood by microscopy

c Placental malaria detected in placental tissue by histology

50 [17], 257 [34], and 400 [19, 21]. The duration of follow-up of infants ranged from 1 to 5 years. In 10 studies, infants were followed up from birth to 1 year of age [16, 18-21, 34-38]. Maternal malaria exposure was determined by (1) microscopy $(\mathrm{N}=5)[18,36,37,39]$ or DNA PCR of maternal blood [40], (2) microscopy of placental blood only $(\mathrm{N}=6)[17,18,21,34,35,37]$, or (3) histology of placental tissue $(\mathrm{N}=5)[16,19,36,40,41]$. Malaria infection during infancy was measured as malaria parasitaemia (prevalence of parasitaemia, $\mathrm{N}=7$; time to first parasitaemia, $\mathrm{N}=4$ ), and clinical malaria (incidence of clinical malaria, $\mathrm{N}=5$; time to first clinical malaria, $\mathrm{N}=2$ ). Malaria parasitaemia was assessed during weekly [35], fortnightly [21], or monthly [18, 37] routine visits. Clinical malaria was assessed by active surveillance in three studies $[21,35,36]$ and by passive surveillance in 11 of the studies [16-20,34, 37-41]. ITN use in infants was not reported in half of the studies. In five studies, ITN use was reported as $100 \%$ [20, 41], 51\% [21], 73\% [19], and $95 \%$ [18]. In one study, $66 \%$ of infants were from families that owned at least an ITN at enrolment [35] while in another study, all infants did not have ITNs [34]. The 
median number of infants born to mothers with maternal parasitaemia was 224 (range 28-236). The median number of infants born to mothers with PM detected by microscopy or histology was 59 (range 15-445). Out of 11 studies which assessed association between PM and the risk of malaria in infants, there were three studies with $\geq 100$ infants born to mothers with PM.

\section{Association between maternal parasitaemia and the risk of malaria in infancy}

The association between maternal parasitaemia and malaria risk in infancy was assessed in three cohort studies [18, 37, 39] and one RCT of IPTp-SP vs IPTp with chloroquine (CQ) vs CQ prophylaxis conducted in Malawi [40] (Table 2). In Malawi, infants born to mothers with malaria parasitaemia detected during the 2nd or 3rd trimester had higher odds of parasitaemia compared to infants born to mothers without parasitaemia during pregnancy, but the difference was not statistically significant [40]. In a cohort study conducted in Uganda, infants born to mothers with any parasitaemia detected by microscopy during pregnancy had a higher risk of parasitaemia during the first year of life compared to infants born to mothers without parasitaemia during pregnancy adjusted for gravidity, birth season, haemoglobin genotype, and residence (risk ratio [RR] 2.97; 95\% confidence interval [CI] 1.37-6.42) [37]. In Benin [18], infants born to mothers having parasitaemia in the 3rd trimester had higher prevalence of parasitaemia (odds ratio [OR] 4.16; 95\% CI 1.64-10.54), shorter time to first clinical malaria (hazard ratio [HR] 3.19; 95\% CI 1.59-6.38) and shorter time to first parasitaemia (HR 2.95; 95\% CI 1.58-5.5) compared to those born to mothers without parasitaemia in the 3rd trimester after adjusting for birth season and residence near the lake (Table 2). In the same study, maternal parasitaemia detected during 1st or 2nd trimester was not associated with an increased risk of malaria during infancy [18]. In another cohort study conducted in Uganda, infants born to mothers with malaria parasitaemia at enrolment or at delivery had a higher incidence of clinical malaria during the first 5 years of life compared to infants born to mothers without parasitaemia (HR 1.23; 95\% CI 1.01-1.51) [39].

\section{Association between placental malaria and risk of malaria in infants}

Six studies evaluating the association between PM detected by microscopy only and the risk of malaria in infants produced mixed results (Table 3 ). The prevalence of parasitaemia was higher in infants born to mothers with PM detected by microscopy compared to those born to mothers without PM (RR 10.42; 95\% CI 2.64-41.10) in a cohort study conducted in Uganda [37] while the prevalence of parasitaemia and clinical malaria tended to be lower in Benin [18] though this was not statistically significant (OR 0.72; 95\% CI 0.25-2.11). Compared to infants born to mothers without PM, infants born to mothers with PM detected by microscopy had a shorter time to first parasitaemia in Cameroon [34], Benin [35] and Tanzania [21], and a shorter time to first clinical malaria in Gabon (HR 2.1; 95\% CI 1.2-3.7) [17]. The only study that adjusted for malaria exposure among other confounding factors showed an association between PM detected by microscopy and time to first parasitaemia but only among infants resident in houses with ITNs (HR 2.13; 95\% CI 1.24-3.67) [35]. In another study conducted in Benin, there was no statistically significant association between PM detected by microscopy and time to first parasitaemia (OR 0.68 ; 95\% CI $0.34-1.38$ ) or time to first clinical malaria (HR 0.60; 95\% CI 0.28-1.32) in infants [18].

Five studies evaluated associations between PM detected by histology and the risk of malaria in infancy (Table 4). Histology detected PM was associated with an increase in the odds of clinical malaria in Malawi (OR 3.9; 95\% CI 1.2-13.0) [40], Tanzania (OR 4.79; 95\% 2.2110.38) [41], and Mozambique (OR 4.63; 95\% CI 2.1010.24) [16] while one study in Cameroon did not find a statistically significant association between histologically detected PM and prevalence of malaria in infants (OR 0.72; 95\% CI 0.40-1.28) [36]. Histologically detected PM was associated with a higher incidence of malaria in Malawi (unadjusted incident rate ratio [IRR] 2.3; 95\% CI 1.1-4.8) [40], but this was not observed in Ghana (IRR 0.86; 95\% CI 0.54-1.37) [19].

\section{Impact of IPTp on the risk of malaria in infants}

Four studies evaluated the impact of IPTp on the risk of malaria in infants (Table 5). Of these, three were RCTs and one was an observational study where some women received IPTp-SP and others received no IPTp. There was no significant difference in the incidence of malaria among infants born to mothers randomized to IPTp-MQ vs IPTp-SP (IRR 0.95; 95\% CI 0.81-1.13) in a multicentre RCT conducted in Benin, Gabon, Tanzania, and Mozambique [38] and among infants born to mothers randomized to intermittent screening and treatment of MiP (ISTp) with artemether-lumefantrine (AL) vs IPTp-SP (IRR 0.94; 95\% CI 0.68-1.59) conducted in Ghana [19]. In a cohort study conducted in Ghana, the risk of malaria was higher in infants born to mothers who did not receive IPTp compared to infants born to mothers who received IPTp-SP, but this difference was also not statistically significant [20]. In Mozambique, the odds of clinical malaria in infants born to mothers who were randomized to IPTp-SP were higher, but not statistically significantly 
Table 2 Association between maternal parasitaemia and malaria risk in infancy stratified by outcome measure

\begin{tabular}{|c|c|c|c|c|c|}
\hline $\begin{array}{l}\text { Author, year } \\
\text { of publication (refs.) }\end{array}$ & $\begin{array}{l}\text { Timing } \\
\text { of measurement } \\
\text { of maternal } \\
\text { parasitaemia }\end{array}$ & $\begin{array}{l}\text { Method used } \\
\text { to detect maternal } \\
\text { parasitaemia }\end{array}$ & Exposure groups (n) & $\begin{array}{l}\text { Measure } \\
\text { of association }(95 \% \\
C \mathrm{Cl}), p \text { value }\end{array}$ & $\begin{array}{l}\text { Confounders adjusted } \\
\text { for }\end{array}$ \\
\hline \multicolumn{6}{|c|}{ Prevalence of parasitaemia } \\
\hline \multirow[t]{2}{*}{ Boudova, 2017 [40] } & \multirow[t]{2}{*}{ 2nd and 3rd trimester } & \multirow[t]{2}{*}{$P C R$} & Unexposed $(n=184)$ & Reference & \multirow{2}{*}{$\begin{array}{l}\text { Maternal age, gestation } \\
\text { age at delivery, IPTp } \\
\text { arm }\end{array}$} \\
\hline & & & Exposed $(n=28)$ & $\begin{aligned} \mathrm{OR} & =1.5(0.5-4.4) \\
\mathrm{P} & =0.45\end{aligned}$ & \\
\hline \multirow{2}{*}{$\begin{array}{l}\text { De Beaudrap, } 2016 \\
\text { [37] }\end{array}$} & \multirow[t]{2}{*}{ 2nd and 3rd trimester } & \multirow[t]{2}{*}{ Microscopy or RDT } & Unexposed $(n=626)$ & Reference & \multirow{2}{*}{$\begin{array}{l}\text { Gravidity, birth season, } \\
\text { haemoglobin geno- } \\
\text { type, residence }\end{array}$} \\
\hline & & & Exposed $(n=198)$ & $\begin{aligned} R R & =2.97(1.37-6.42), \\
p & =N R\end{aligned}$ & \\
\hline \multirow[t]{6}{*}{ Borgella, 2013 [18] } & \multirow[t]{2}{*}{ 1st trimester } & \multirow[t]{2}{*}{ Microscopy } & Unexposed $(n=N A)$ & Reference & \multirow{6}{*}{$\begin{array}{l}\text { Residence near the lake, } \\
\text { birth season }\end{array}$} \\
\hline & & & Exposed $(n=N A)$ & $\begin{aligned} \mathrm{OR} & =1.12(0.23-5.45) \\
\mathrm{p} & =0.89\end{aligned}$ & \\
\hline & \multirow[t]{2}{*}{ 2nd trimester } & \multirow[t]{2}{*}{ Microscopy } & Unexposed $(n=142)$ & Reference & \\
\hline & & & Exposed $(n=52)$ & $\begin{aligned} O R & =0.87(0.35-2.09) \\
p & =0.75\end{aligned}$ & \\
\hline & \multirow[t]{2}{*}{ 3rd trimester } & \multirow[t]{2}{*}{ Microscopy } & Unexposed $(n=121)$ & Reference & \\
\hline & & & Exposed $(n=73)$ & $\begin{aligned} \mathrm{OR} & =4.16(1.64-10.54) \\
\mathrm{P} & =0.003\end{aligned}$ & \\
\hline \multicolumn{6}{|c|}{ Time to first parasitaemia } \\
\hline \multirow[t]{6}{*}{ Borgella, 2013 [18] } & \multirow[t]{2}{*}{ 1st trimester } & \multirow[t]{2}{*}{ Microscopy } & Unexposed $(n=N A)$ & Reference & \multirow{6}{*}{$\begin{array}{l}\text { Residence near the lake, } \\
\text { birth season }\end{array}$} \\
\hline & & & Exposed $(n=N A)$ & $\begin{aligned} H R & =1.00(0.42-2.39) \\
p & =0.99\end{aligned}$ & \\
\hline & \multirow[t]{2}{*}{ 2nd trimester } & \multirow[t]{2}{*}{ Microscopy } & Unexposed $(n=142)$ & Reference & \\
\hline & & & Exposed $(n=52)$ & $\begin{aligned} H R & =1.14(0.62-2.12) \\
p & =0.68\end{aligned}$ & \\
\hline & \multirow[t]{2}{*}{ 3rd trimester } & \multirow[t]{2}{*}{ Microscopy } & Unexposed $(n=121)$ & Reference & \\
\hline & & & Exposed $(n=73)$ & $\begin{aligned} H R & =2.95(1.58-5.50) \\
p & =0.001\end{aligned}$ & \\
\hline \multicolumn{6}{|c|}{ Time to first clinical malaria } \\
\hline \multirow[t]{6}{*}{ Borgella, 2013 [18] } & \multirow[t]{2}{*}{ 1st trimester } & \multirow[t]{2}{*}{ Microscopy } & Unexposed $(n=N A)$ & Reference & \multirow{6}{*}{$\begin{array}{l}\text { Residence near the lake, } \\
\text { birth season }\end{array}$} \\
\hline & & & Exposed $(n=N A)$ & $\begin{aligned} H R & =0.97(0.32-2.92) \\
p & =0.95\end{aligned}$ & \\
\hline & \multirow[t]{2}{*}{ 2nd trimester } & Microscopy & Unexposed $(n=142)$ & Reference & \\
\hline & & & Exposed $(n=52)$ & $\begin{aligned} H R & =1.15(0.58-2.28) \\
p & =0.70\end{aligned}$ & \\
\hline & 3rd trimester & Microscopy & Unexposed $(n=121)$ & Reference & \\
\hline & & & Exposed $(n=73)$ & $\begin{aligned} H R & =3.19(1.59-6.38) \\
p & =0.001\end{aligned}$ & \\
\hline Incidence of clinical ma & aria & & & & \\
\hline Ndibazza, 2013 [39] & Baseline and delivery & Microscopy & Unexposed $(n=2053)$ & Ref & Maternal age, ITN pos- \\
\hline & & & Exposed $(n=236)$ & $\begin{aligned} H R & =1.23(1.01-1.51) \\
p & =0.04\end{aligned}$ & $\begin{array}{l}\text { session, parity, educa- } \\
\text { tion, social economic } \\
\text { status, residence, } \\
\text { mother's HIV status }\end{array}$ \\
\hline
\end{tabular}

Cl confidence interval, HIV human immunodeficiency virus, HR hazard ratio, IPTp intermittent preventive treatment of malaria in pregnancy, ITN insecticide treated net, $N A$ not applicable number was imputed, $N R$ not reported, $O R$ odds ratio, $R D T$ rapid diagnostic test, $R R$ risk ratio, $P C R$ polymerase chain reaction

so, than in infants born to mothers randomized to placebo (OR 1.28; 95\% CI 0.90-1.83) [16].

\section{Assessment of risk of bias in individual studies}

Risk of bias in individual studies was assessed using the Newcastle-Ottawa scale for observational studies
(Table 6) and the Cochrane Collaboration tool for RCTs (Table 7). One study where IPTp was randomized was assessed using the Newcastle-Ottawa Scale for assessing risk of bias in observational studies because the study only assessed an association between MiP (and 
Table 3 Association between placental malaria detected by microscopy and the risk of malaria in infancy stratified by outcome

\begin{tabular}{|c|c|c|c|}
\hline Country, year of publication (ref) & PM exposure group (n) & $\begin{array}{l}\text { Measure of association }(95 \% \mathrm{Cl}) \text {, } \\
\text { p-value }\end{array}$ & Confounders adjusted for \\
\hline \multicolumn{4}{|l|}{ Prevalence of parasitaemia } \\
\hline \multirow[t]{2}{*}{ De Beaudrap, 2016 [37] } & Unexposed (475) & Reference & \multirow{2}{*}{$\begin{array}{l}\text { Gravidity, maternal age, residence, level of } \\
\text { education, season, maternal HIV status, } \\
\text { ITN use }\end{array}$} \\
\hline & Exposed (15) & $\mathrm{RR}=10.42(2.64-41.10), \mathrm{p}=\mathrm{NR}$ & \\
\hline \multirow[t]{2}{*}{ Borgella, 2013 [18] } & Unexposed (154 & Reference & \multirow[t]{2}{*}{ Residence near the lake, birth season } \\
\hline & Exposed (36) & $O R=0.72(0.25-2.11), p=0.55$ & \\
\hline \multicolumn{4}{|l|}{ Time to first parasitaemia } \\
\hline \multirow[t]{3}{*}{ Tassi Yunga, $2018^{a}$ [34] } & No PM (36) & Reference & \multirow{3}{*}{$\begin{array}{l}\text { Gravidity, birth season Hb genotype, } \\
\text { residence }\end{array}$} \\
\hline & PM Lo (18) & $H R=2.6(1.3-4.8)$ & \\
\hline & PM Hi (18) & $\mathrm{HR}=1.5(0.7-3.7)$ & \\
\hline \multirow[t]{2}{*}{ Borgella, 2013 [18] } & Unexposed (154) & Reference & \multirow[t]{2}{*}{ Residence near the lake, birth season } \\
\hline & Exposed (36) & $H R=0.68(0.34-1.38), p=0.29$ & \\
\hline \multirow[t]{2}{*}{ Le Port, 2011 [35] } & Unexposed (485) & Reference & \multirow[t]{2}{*}{ Unadjusted } \\
\hline & Exposed (59) & $H R=1.62(1.08-2.43), p=0.02$ & \\
\hline \multirow[t]{4}{*}{ Le Port, 2011 [35] } & Unexposed, had ITN (321) & Reference & \multirow{4}{*}{$\begin{array}{l}\text { Exposure to anopheles, season, antenatal } \\
\text { care, severe anaemia }\end{array}$} \\
\hline & Exposed, had ITN (34) & $H R=2.13(1.24-3.67), p<0.01$ & \\
\hline & Unexposed, no ITN (158) & Reference & \\
\hline & Exposed, no ITN (25) & $H R=1.18(0.60-2.33), p=0.62$ & \\
\hline \multirow[t]{2}{*}{ Mutabingwa, 2005 [21] } & Unexposed (384) & Reference & \multirow{2}{*}{$\begin{array}{l}\text { Gravidity, residence, transmission season } \\
\text { at birth, and bed net usage }\end{array}$} \\
\hline & Exposed (69) & $H R=1.41(1.01-1.99), p=N R$ & \\
\hline \multicolumn{4}{|l|}{ Time to first clinical malaria } \\
\hline \multirow[t]{2}{*}{ Borgella, 2013 [18] } & Unexposed (154) & Reference & \multirow[t]{2}{*}{ Residence near the lake, birth season } \\
\hline & Exposed (36) & $H R=0.60(0.28-1.32), p=0.21$ & \\
\hline \multirow[t]{2}{*}{ Schwarz, 2008 [17] } & Unexposed (477) & Reference & \multirow{2}{*}{$\begin{array}{l}\text { Gravidity, residence, birth season, IPTi, bec } \\
\text { net use }\end{array}$} \\
\hline & Exposed (50) & $H R=2.1(1.2-3.7), p=N R$ & \\
\hline
\end{tabular}

Cl confidence interval, HIV human immunodeficiency virus, HR hazard ratio, IPTi intermittent preventive treatment of malaria in infancy, ITN insecticide treated net, NR Not reported, $O R$ odds ratio, $P M$ placental malaria, $R R$ risk ratio

a Placental malaria detected by microscopy or PCR; PM Lo, placental malaria with $<25$ infected erythrocytes/ $\mu \mathrm{L} ; \mathrm{PM} \mathrm{Hi}$, placental malaria with $>25$ infected erythrocytes/ $\mu \mathrm{L}$

not IPTp regimens) and the risk of clinical malaria or parasitaemia in the infant [40]. All cohort studies had a representative exposed cohort, selected the nonexposed comparison group adequately and measured malaria exposure during pregnancy, and demonstrated that the outcome was not present at the beginning of follow-up. Most of the studies adjusted for IPTp and ITN use but only two studies [20,35] adjusted for malaria exposure. In three of the studies, the number of participants lost to follow-up or the reasons for losses to follow-up were not reported [16, 36, 41]. Three studies had $>20 \%$ losses to follow-up $[17,36,40]$. The overall risk of bias in cohort studies was rated as high in nine studies [17, 18, 21, 34, 36, 37, 39-41] and medium in two studies $[20,35]$. In all the RCTs, allocation concealment was adequate, and no trial was stopped before completion. Overall, the risk of bias in all three RCTs was low.

\section{Discussion}

This systematic review assessed evidence evaluating the associations between MiP or IPTp and the risk of malaria infection or illness during infancy. Overall, the available evidence is of insufficient quality to confirm or rule out an association between maternal malaria infection and the risk of malaria during infancy. Most studies had small numbers of exposed infants and failed to control for possible confounding by malaria transmission intensity shared between mothers and their infants. Only one study that examined the association between malaria in pregnancy and the risk of malaria in infants controlled for malaria transmission at the level of the household [35]. In this study, time to first parasitaemia in infants born to mothers with PM was shorter in those who lived in households with ITNs, than in those living in households without ITNs. It is possible that household use of ITNs reflects underlying malaria transmission intensity, with households exposed to higher transmission more likely to 
Table 4 Association between placental malaria detected by histology and the risk of malaria in infancy stratified by outcome

\begin{tabular}{|c|c|c|c|}
\hline $\begin{array}{l}\text { Country, year } \\
\text { of publication (ref) }\end{array}$ & $\begin{array}{l}\text { Placental malaria } \\
\text { exposure group }(n)\end{array}$ & Measure of association $(95 \% \mathrm{Cl})$, p-value & Confounders adjusted for \\
\hline \multicolumn{4}{|l|}{ Clinical malaria } \\
\hline \multirow[t]{2}{*}{ Boudova, 2017 [40] } & Unexposed (184) & Reference & Maternal age, gestation age at delivery, IPTp arm \\
\hline & Exposed (67) & $\mathrm{OR}=3.9(1.2-13.0), p=0.03$ & \\
\hline \multirow[t]{2}{*}{ Boudova, 2017 [40] } & Unexposed (184) & Reference & Unadjusted \\
\hline & Exposed (67) & $\mathrm{IRR}=2.3(1.1-4.8), \mathrm{p}=\mathrm{NR}$ & \\
\hline \multirow[t]{2}{*}{ Sylvester, 2016 [41] } & Unexposed (165) & Reference & Gravity, season of birth, infant birth weight, maternal age \\
\hline & Exposed (41) & $\mathrm{OR}=4.79(2.21-10.38), \mathrm{p}<0.05$ & \\
\hline \multirow[t]{2}{*}{ Awine, 2016 [19] } & Unexposed (484) & Reference & ITN use, gender, social economic status, living near an \\
\hline & Exposed (202) & $\operatorname{IRR}=0.86(0.54-1.37), p=0.52$ & $\begin{array}{l}\text { irrigated area, infant age, maternal baseline parasitae- } \\
\text { mia }\end{array}$ \\
\hline \multirow[t]{2}{*}{ Apinjoh, 2015 [36] } & Unexposed $(n=237)$ & Reference & Not indicated \\
\hline & Exposed $(n=166)$ & $O R=0.72(0.40-1.28), p=0.26$ & \\
\hline \multirow[t]{4}{*}{ Bardaji, 2011 [16] } & Unexposed (424) & Reference & Residence near the lake, birth season \\
\hline & Past infection (321) & $\mathrm{OR}=3.06(1.94-4.82), \mathrm{p}<0.001$ & \\
\hline & Acute infection (42) & $\mathrm{OR}=4.63(2.10-10.24), \mathrm{p}<0.001$ & \\
\hline & Chronic infection (82) & $\mathrm{OR}=3.95(2.07-10.24), \mathrm{p}<0.001$ & \\
\hline \multicolumn{4}{|c|}{ Prevalence of parasitaemia } \\
\hline \multirow[t]{2}{*}{ Boudova, 2017 [40] } & Unexposed (184) & Reference & Maternal age, gestation age at delivery, IPTp arm \\
\hline & Exposed (67) & $\mathrm{OR}=2.5(1.0-6.3), p=0.06$ & \\
\hline
\end{tabular}

$C I$ confidence interval, IPTp intermittent preventive treatment of malaria in pregnancy, IRR, incident rate ratio, ITN insecticide treated net, NR not reported, $O R$ odds ratio

Table 5 Impact of IPTp on the risk of malaria in infancy

\begin{tabular}{|c|c|c|c|c|}
\hline Author, year of publication & $\begin{array}{l}\text { Randomized } \\
\text { Y/N }\end{array}$ & IPTp arm (n) & $\begin{array}{l}\text { Measure of association }(95 \% \\
\mathrm{Cl}), \mathrm{p} \text {-value }\end{array}$ & Confounders adjusted for \\
\hline \multirow[t]{2}{*}{ Ruperez, 2016 [38] } & \multirow[t]{2}{*}{ Yes } & IPTp-SP (1432) & Reference & \multirow[t]{2}{*}{ Country } \\
\hline & & IPTP-MQ (2815) & $\operatorname{IRR}=0.95(0.81-1.13), p=0.60$ & \\
\hline \multirow[t]{2}{*}{ Awine, 2016 [19] } & \multirow[t]{2}{*}{ Yes } & IPTp-SP (495) & Reference & \multirow{2}{*}{$\begin{array}{l}\text { Gender, social economic status, residence, irrigated } \\
\text { area, season, ITN use, baseline parasitaemia, } \\
\text { maternal haemoglobin }\end{array}$} \\
\hline & & ISTp-AL (493) & $\mathrm{IRR}=0.94(0.68-1.59), p=0.76$ & \\
\hline \multirow[t]{2}{*}{ Asante, 2013 [20] } & \multirow[t]{2}{*}{ No } & IPTp-SP (1755) & Reference & \multirow[t]{2}{*}{ Unadjusted } \\
\hline & & No IPTp (97) & $H R=1.23(0.93-1.59), p=0.15$ & \\
\hline \multirow[t]{2}{*}{ Bardaji, 2011 [16] } & \multirow[t]{2}{*}{ Yes } & Placebo (500) & Reference & \multirow[t]{2}{*}{ Unadjusted } \\
\hline & & IPTp-SP (497) & $\mathrm{OR}=1.28(0.90-1.83), p=0.17$ & \\
\hline
\end{tabular}

$A L$ artemether lumefantrine, $C l$ confidence interval, IPTp intermittent preventive treatment of malaria in pregnancy, IRR incident rate ratio, ISTp intermittent screening and treatment of malaria in pregnancy, ITN insecticide treated nets, HR hazard ratio, MQ mefloquine, SP sulfadoxine-pyrimethamine

use ITNs, and mothers and infants in such households at higher risk of malaria. However, secondary data analysis of the study did not find an association between PM and the risk of subsequent clinical malaria episodes [42]. This suggests that the effect of PM on the risk of malaria in infants wanes over time.

Majority of the studies included in this review showed an increased risk of clinical malaria or parasitaemia in infants born to mothers with maternal peripheral parasitaemia [37, 39], infants born to mothers with
PM detected by microscopy [17, 21, 34, 35, 37] and in infants born to mothers with PM detected by histology $[16,40,41]$. These results could possibly be explained by confounding due to differences in malaria transmission intensity. Infants born to mothers with maternal peripheral parasitaemia during pregnancy or PM at delivery could be at higher risk of clinical malaria or parasitaemia because they live in an environment with higher risk of malaria transmission just like their mothers [43]. 
Table 6 Assessment of risk of bias for observational studies using the Newcastle-Ottawa scale

\begin{tabular}{|c|c|c|c|c|c|c|c|c|c|c|c|}
\hline \multirow[t]{2}{*}{ Author, year of publication (ref) } & \multicolumn{4}{|c|}{ Selection } & \multicolumn{2}{|c|}{ Comparability } & \multicolumn{3}{|c|}{ Outcome } & \multirow[t]{2}{*}{ Total } & \multirow[t]{2}{*}{ Overall risk of bias } \\
\hline & REC & SNEC & ME & DON & AME & Al & AO & $\mathrm{FL}$ & CF & & \\
\hline Tassi Yunga, 2018 [34] & * & $*$ & * & * & - & - & $*$ & * & * & 7 & High \\
\hline Boudova, 2017 [40] & * & * & * & * & - & * & * & * & - & 7 & High \\
\hline Sylvester, 2016 [41] & * & * & * & * & - & - & * & * & - & 6 & High \\
\hline De Beaudrap, 2016 [37] & * & * & * & * & - & * & * & * & * & 8 & High \\
\hline Apinjoh, 2015 [36] & * & $*$ & * & * & - & - & * & * & - & 6 & High \\
\hline Ndibazza, 2013 [39] & * & $*$ & $*$ & * & - & $*$ & $*$ & $*$ & * & 8 & High \\
\hline Borgella, 2013 [18] & $*$ & $*$ & $*$ & $*$ & - & * & $*$ & $*$ & * & 8 & High \\
\hline Asante, 2013 [20] & * & * & * & * & * & * & * & * & * & 9 & Medium \\
\hline Le Port, 2011 [35] & * & * & $*$ & * & * & * & $*$ & * & * & 9 & Medium \\
\hline Schwarz, 2008 [17] & * & * & * & * & - & * & $*$ & * & - & 7 & High \\
\hline Mutabingwa, 2005 [21] & * & * & * & * & - & * & * & * & * & 8 & High \\
\hline
\end{tabular}

REC representativeness of the exposed cohort, SNEC selection of the non-exposed cohort ME measurement of exposure to malaria during pregnancy, DON demonstration that the outcome of interest was not present at the start of the study, AME adjusted for malaria transmission exposure $A /$ adjusted for IPTp or insecticide treated net use, $A O$ assessment of the outcome $F L$ follow-up long enough for outcome to occur, $C F$ completeness of follow-up

- , score of zero; ${ }^{*}$, score of one

Table 7 Assessment of risk of bias in randomized trials comparing the risk of malaria among infants who received different IPTp regimens

\begin{tabular}{llll}
\hline Criterion & Studies & \\
\cline { 2 - 3 } & Ruperez, 2016 [38] & Awine, 2016 [19] & Bardaji, \\
$\mathbf{2 0 1 1}$ & \\
[16] & Yes \\
\hline Allocation concealment & & No & Yes \\
Trial stopped early & Yes & No & No \\
Participants blinded & No & No & Yes \\
Study staff blinded & No & Yes & Yes \\
Infant malaria assessed blinded & No & NR & Yes \\
Proportion of infants lost to follow-up & Yes & Low & NR \\
Overall risk of bias & $972 / 4247(22.9 \%)$ & Low \\
\hline
\end{tabular}

NR not reported

Because it would not be ethical and feasible to randomize pregnant women to exposure to MiP, alternative study designs are needed, such as randomising pregnant women to IPTp interventions with different efficacies and comparing the risks of parasitaemia and clinical malaria in the different sets of infants born to mothers who received different IPTp interventions. In this systematic review, three RCTs which evaluated the association between IPTp and the risk of malaria during infancy showed no difference in the risk of malaria among infants born to mothers who received IPTpMQ vs IPTp-SP [38], ISTp-AL vs IPTp-SP [19], and IPTp-SP vs placebo [16]. These studies were possibly limited by the failure of the alternative intervention to significantly reduce the burden of malaria especially
PM during pregnancy $[44,45]$. The prevalence of PM was not significantly different among mothers randomized to IPTp-MQ (4.6\%) compared to IPTp SP $(5.4 \%$, $\mathrm{p}=0.19)$ in the multicentre trial [44], and was similar among mothers randomized to IPTp-SP (24.5\%) compared to ISTp-AL (24.2\%) in the Ghana trial [45]. In the Mozambique trial, the prevalence of any PM detected by histology or microscopy was similar among mothers on IPTp-SP (52\%) or placebo (52\%) [46]. Although in the same trial, the prevalence of PM detected by microscopy was higher among women on placebo (14\%) compared to women on IPTp-SP (7\%) [46], the risk of clinical malaria did not differ among infants born to mothers on the two IPTp arms [16]. This could possibly be due to few malaria outcomes during infancy 
(there were 135 first clinical malaria episodes during follow-up), which limited the power of the study.

There is currently a promising alternative drug combination for IPTp, which substantially reduces the burden of malaria during pregnancy including PM compared to SP. Although SP remains the drug recommended by the WHO for IPTp [22], its effectiveness is affected by widespread antifolate resistance [47]. In East Africa, IPTpDP has been shown to markedly reduce the incidence of clinical malaria and the prevalence of parasitaemia during pregnancy, and the prevalence of PM at delivery compared to IPTp-SP $[25,26,29]$. One study has evaluated the impact of IPTp-DP on malaria during infancy in Uganda. In this randomized controlled trial, which examined infants receiving DP for malaria prevention, the incidence of malaria during the first 2 years of life was higher in infants born to pregnant women who received IPTp-DP (given monthly) than in those born to women who received IPTp-SP (given every 2 months). This effect was magnified in female infants [48]. The reason for this finding is unclear, but may be due to lower blood levels of piperaquine, which were observed in female infants born to mothers who received IPTp-DP [48]. Lower piperaquine levels have been associated with a higher risk of malaria in children taking DP for malaria prevention [49], but the reason why female infants born to mothers receiving IPTp-DP would have lower levels of piperaquine is unknown.

Several studies have suggested immune tolerance [9, $12,14,50]$ as one of the potential mechanisms for the observed association between MiP and the risk of malaria in infants. Evidence from laboratory studies shows that in utero exposure to malaria antigens is associated with a bias of fetal immune responses to $P$. falciparum specific [12-14] or non-malaria specific [12, 50] antigens towards anti-inflammatory responses suggesting that exposure to malaria in utero may not only affect development of malaria specific immunity in the fetus but may also affect non-malaria specific immunity. Indeed, one study has reported an increased risk of non-malaria febrile illnesses in infants born to mothers with PM compared to infants born to mothers without PM [51]. Also, PM has been associated with a reduced maternal-fetal transfer of antibodies to $P$. falciparum [52, 53], but this was not associated with an increased risk of malaria in infants $[52,53]$.

This systematic review had several limitations. Substantial heterogeneity in included studies was found. Studies varied in the duration of follow-up, detection of malaria exposure during pregnancy, and approaches to measuring the outcome in infants and to data analysis. Also, important raw data like the number of malaria episodes in each malaria exposure group during pregnancy was not presented in majority of the studies. Majority of studies included in this systematic review used PM as a proxy measure of MiP. PM may not be a good proxy measure of MiP because some mothers with peripheral malaria parasitaemia may clear the parasites especially with highly efficacious IPTp drugs. However, majority of the included studies used IPTp-SP, which is not highly effective at clearing parasites [54]. Only studies published in English were included in this systematic review. This could have led to missing out on potential studies published in English. However, a literature search not limiting the language to English did not yield any such studies. The search strategy was also limited to only published studies. This could have limited the number of studies with null findings, which are less likely to be published.

\section{Conclusion}

The results of this systematic review suggest that there is insufficient evidence to confirm or exclude a causal association between $\mathrm{MiP}$ and the risk of malaria during infancy. Also, evidence on the impact of IPTp on the risk of malaria in infancy is inconclusive. There is need to better understand the association between MiP and malaria in infants in order to minimize the effects of MiP on malaria during infancy. Future studies of new IPTp interventions should consider not only evaluating the impact of IPTp on birth outcomes but also the potential impact of the intervention on the risk of clinical malaria or parasitaemia in infancy. This could have important policy implications on the choice of future drugs for IPTp.

\section{Acknowledgements \\ We are grateful to authors of studies included in this systematic review.}

Authors' contributions

All authors contributed to preparing the systematic review protocol. AK did the electronic search of the literature, AK and DC extracted data. All authors participated in the writing and revision of the manuscript. All authors read and approved the final manuscript.

\section{Funding \\ This systematic review was part of the first author's Ph.D. studies which were funded by the Fogarty International Center.}

Availability of data and materials

Not applicable.

Ethics approval and consent to participate

Not applicable.

Consent for publication

Not applicable.

Competing interests

The authors declare that they have no competing interests.

\section{Author details}

${ }^{1}$ Infectious Diseases Research Collaboration, P.O Box 7475, Kampala, Uganda. ${ }^{2}$ London School of Hygiene and Tropical Medicine, Keppel Street, London WC1E 7HT, UK. ${ }^{3}$ University of California San Francisco, San Francisco, CA, USA. ${ }^{4}$ Department of Medicine at the Doherty Institute, University of Melbourne, 792 Elizabeth Street, Melbourne, VIC 3000, Australia. 
Received: 20 May 2019 Accepted: 28 August 2019

Published online: 03 September 2019

\section{References}

1. Dellicour S, Tatem AJ, Guerra CA, Snow RW, ter Kuile FO. Quantifying the number of pregnancies at risk of malaria in 2007: a demographic study. PLoS Med. 2010;7:e1000221.

2. Desai M, ter Kuile FO, Nosten F, McGready R, Asamoa K, Brabin B, et al. Epidemiology and burden of malaria in pregnancy. Lancet Infect Dis. 2007;7:93-104.

3. Omer SA, Idress HE, Adam I, Abdelrahim M, Noureldein AN, Abdelrazig $A M$, et al. Placental malaria and its effect on pregnancy outcomes in Sudanese women from Blue Nile State. Malar J. 2017;16:374.

4. Guyatt HL, Snow RW. Impact of malaria during pregnancy on low birth weight in sub-Saharan Africa. Clin Microbiol Rev. 2004;17:760-9.

5. Guyatt HL, Snow RW. The epidemiology and burden of Plasmodium falciparum-related anemia among pregnant women in sub-Saharan Africa. Am J Trop Med Hyg. 2001;64:36-44.

6. Moore KA, Simpson JA, Scoullar MJL, McGready R, Fowkes FJI. Quantification of the association between malaria in pregnancy and stillbirth: a systematic review and meta-analysis. Lancet Glob Health. 2017;5:e1101-12.

7. Steketee RW, Wirima JJ, Hightower AW, Slutsker L, Heymann DL, Breman JG. The effect of malaria and malaria prevention in pregnancy on offspring birthweight, prematurity, and intrauterine growth retardation in rural Malawi. Am J Trop Med Hyg. 1996;55:33-41.

8. Dauby N, Goetghebuer T, Kollmann TR, Levy J, Marchant A. Uninfected but not unaffected: chronic maternal infections during pregnancy, fetal immunity, and susceptibility to postnatal infections. Lancet Infect Dis. 2012;12:330-40.

9. Brustoski K, Moller U, Kramer M, Petelski A, Brenner S, Palmer DR, et al. IFN-gamma and IL-10 mediate parasite-specific immune responses of cord blood cells induced by pregnancy-associated Plasmodium falciparum malaria. J Immunol. 2005;174:1738-45.

10. Malhotra I, Mungai P, Muchiri E, Ouma J, Sharma S, Kazura JW, et al. Distinct Th1- and Th2-Type prenatal cytokine responses to Plasmodium falciparum erythrocyte invasion ligands. Infect Immun. 2005;73:3462-70.

11. Metenou S, Suguitan AL Jr, Long C, Leke RGF, Taylor DW. Fetal immune responses to Plasmodium falciparum antigens in a malaria-endemic region of Cameroon. J Immunol. 2007;178:2770-7.

12. Bisseye C, van der Sande M, Morgan WD, Holder AA, Pinder M, Ismaili J. Plasmodium falciparum infection of the placenta impacts on the Thelper type 1 (Th1)/Th2 balance of neonatal T cells through CD4(+)CD25(+) forkhead box P3(+) regulatory T cells and interleukin-10. Clin Exp Immunol. 2009:158:287-93.

13. Ismaili J, Van Der Sande M, Holland MJ, Sambou I, Keita S, Allsopp C, et al. Plasmodium falciparum infection of the placenta affects newborn immune responses. Clin Exp Immunol. 2003;133:414-21.

14. Brustoski K, Moller U, Kramer M, Hartgers FC, Kremsner PG, Krzych U, et al. Reduced cord blood immune effector-cell responsiveness mediated by CD4+ cells induced in utero as a consequence of placental Plasmodium falciparum infection. J Infect Dis. 2006;193:146-54.

15. Engelmann I, Santamaria A, Kremsner PG, Luty AJ. Activation status of cord blood gamma delta T cells reflects in utero exposure to Plasmodium falciparum antigen. J Infect Dis. 2005;191:1612-22.

16. Bardaji A, Sigauque B, Sanz S, Maixenchs M, Ordi J, Aponte JJ, et al. Impact of malaria at the end of pregnancy on infant mortality and morbidity. J Infect Dis. 2011;203:691-9.

17. Schwarz NG, Adegnika AA, Breitling LP, Gabor J, Agnandji ST, Newman RD, et al. Placental malaria increases malaria risk in the first 30 months of life. Clin Infect Dis. 2008;47:1017-25.

18. Borgella S, Fievet N, Huynh BT, Ibitokou S, Hounguevou G, Affedjou J, et al. Impact of pregnancy-associated malaria on infant malaria infection in southern Benin. PLoS One. 2013:8:e80624

19. Awine T, Belko MM, Oduro AR, Oyakhirome S, Tagbor H, Chandramohan $D$, et al. The risk of malaria in Ghanaian infants born to women managed in pregnancy with intermittent screening and treatment for malaria or intermittent preventive treatment with sulfadoxine/pyrimethamine. Malar J. 2016:15:46
20. Asante KP, Owusu-Agyei S, Cairns M, Dodoo D, Boamah EA, Gyasi R, et al. Placental malaria and the risk of malaria in infants in a high malaria transmission area in Ghana: a prospective cohort study. J Infect Dis. 2013;208:1504-13.

21. Mutabingwa TK, Bolla MC, Li JL, Domingo GJ, Li X, Fried M, et al. Maternal malaria and gravidity interact to modify infant susceptibility to malaria. PLoS Med. 2005;2:e407.

22. WHO policy brief for the implementation of intermittent preventive treatment of malaria in pregnancy using sulfadoxine-pyrimethamine (IPTp-SP). Geneva: World Health Organization; 2014. http://www.who.int/ malaria/publications/atoz/iptp-sp-updated-policy-brief-24jan2014.pdf.

23. Naidoo I, Roper C. Drug resistance maps to guide intermittent preventive treatment of malaria in African infants. Parasitology. 2011;138:1469-79.

24. van Eijk AM, Larsen DA, Kayentao K, Koshy G, Slaughter DEC, Roper C, et al. Effect of Plasmodium falciparum sulfadoxine-pyrimethamine resistance on the effectiveness of intermittent preventive therapy for malaria in pregnancy in Africa: a systematic review and meta-analysis. Lancet Infect Dis. 2019;19:546-56.

25. Kajubi R, Ochieng T, Kakuru A, Jagannathan P, Nakalembe M, Ruel T, et al. Monthly sulfadoxine-pyrimethamine versus dihydroartemisinin-piperaquine for intermittent preventive treatment of malaria in pregnancy: a double-blind, randomised, controlled, superiority trial. Lancet. 2019;393:1428-39.

26. Kakuru A, Jagannathan $P$, Muhindo MK, Natureeba P, Awori P, Nakalembe $M$, et al. Dihydroartemisinin-piperaquine for the prevention of malaria in pregnancy. N Engl J Med. 2016;374:928-39.

27. Broen K, Brustoski K, Engelmann I, Luty AJ. Placental Plasmodium falciparum infection: causes and consequences of in utero sensitization to parasite antigens. Mol Biochem Parasitol. 2007;151:1-8.

28. Garner P, Gulmezoglu AM. Drugs for preventing malaria in pregnant women. Cochrane Database Syst Rev. 2006;18:000169.

29. Desai M, Gutman J, L'Lanziva A, Otieno K, Juma E, Kariuki S, et al. Intermittent screening and treatment or intermittent preventive treatment with dihydroartemisinin-piperaquine versus intermittent preventive treatment with sulfadoxine-pyrimethamine for the control of malaria during pregnancy in western Kenya: an open-label, three-group, randomised controlled superiority trial. Lancet. 2015;386:2507-19.

30. Shamseer L, Moher D, Clarke M, Ghersi D, Liberati A, Petticrew M, et al. Preferred reporting items for systematic review and meta-analysis protocols (PRISMA-P) 2015: elaboration and explanation. BMJ. 2015;349:g7647.

31. Malaria in Pregnancy Library. http://library.mip-consortium.org/.

32. The Newcastle-Ottawa Scale (NOS) for assessing the quality of nonrandomized studies in metaanalyses. http://www.ohri.ca/programs/clini cal_epidemiology/oxford.asp.

33. Higgins JP, Altman DG, Gotzsche PC, Juni P, Moher D, Oxman AD, et al. The Cochrane Collaboration's tool for assessing risk of bias in randomised trials. BMJ. 2011;343:d5928.

34. Tassi Yunga S, Fouda GG, Sama G, Ngu JB, Leke RGF, Taylor DW. Increased susceptibility to Plasmodium falciparum in infants is associated with low, not high, placental malaria parasitemia. Sci Rep. 2018;8:169.

35. Le Port A, Watier L, Cottrell G, Ouedraogo S, Dechavanne C, Pierrat C, et al. Infections in infants during the first 12 months of life: role of placental malaria and environmental factors. PLOS ONE. 2011:6:e27516.

36. Apinjoh TO, Anchang-Kimbi JK, Mugri RN, Njua-Yafi C, Tata RB, Chi HF, et al. Determinants of infant susceptibility to malaria during the first year of life in South Western cameroon. Open Forum Infect Dis. 2015;2:ofv012.

37. De Beaudrap P, Turyakira E, Nabasumba C, Tumwebaze B, Piola P, Boum li $Y$, et al. Timing of malaria in pregnancy and impact on infant growth and morbidity: a cohort study in Uganda. Malar J. 2016;15:92.

38. Ruperez M, Gonzalez R, Mombo-Ngoma G, Kabanywanyi AM, Sevene E, Ouedraogo S, et al. Mortality, morbidity, and developmental outcomes in infants born to women who received either mefloquine or sulfadoxine-pyrimethamine as intermittent preventive treatment of malaria in pregnancy: a cohort study. PLoS Med. 2016;13:e1001964.

39. Ndibazza J, Webb EL, Lule S, Mpairwe H, Akello M, Oduru G, et al. Associations between maternal helminth and malaria infections in pregnancy and clinical malaria in the offspring: a birth cohort in entebbe, Uganda. J Infect Dis. 2013;208:2007-16.

40. Boudova S, Divala T, Mungwira R, Mawindo P, Tomoka T, Laufer MK. Placental but not peripheral Plasmodium falciparum infection during 
pregnancy is associated with increased risk of malaria in infancy. J Infect Dis. 2017;216:732-5.

41. Sylvester B, Gasarasi DB, Aboud S, Tarimo D, Massawe S, Mpembeni R, et al. Prenatal exposure to Plasmodium falciparum increases frequency and shortens time from birth to first clinical malaria episodes during the first two years of life: prospective birth cohort study. Malar J. 2016;15:379.

42. Bouaziz O, Courtin D, Cottrell G, Milet J, Nuel G, Garcia A. Is placental malaria a long term risk factor for mild malaria attack in infancy? Revisiting a paradigm. Clin Infect Dis. 2018;66:930-5.

43. Cairns M, Gosling R, Chandramohan D. Placental malaria increases malaria risk in the first 30 months of life: not causal. Clin Infect Dis. 2009;48:497-8 (author reply 498-9)

44. Gonzalez R, Mombo-Ngoma G, Ouedraogo S, Kakolwa MA, Abdulla S, Accrombessi $\mathrm{M}$, et al. Intermittent preventive treatment of malaria in pregnancy with mefloquine in HIV-negative women: a multicentre randomized controlled trial. PLoS Med. 2014;11:e1001733.

45. Tagbor H, Cairns M, Bojang K, Coulibaly SO, Kayentao K, Williams J, et al. A non-inferiority, individually randomized trial of intermittent screening and treatment versus intermittent preventive treatment in the control of malaria in pregnancy. PLoS One. 2015;10:e132247.

46. Menendez C, Bardaji A, Sigauque B, Romagosa C, Sanz S, Serra-Casas E, et al. A randomized placebo-controlled trial of intermittent preventive treatment in pregnant women in the context of insecticide treated nets delivered through the antenatal clinic. PLoS One. 2008;3:e1934.

47. Naidoo I, Roper C. Mapping 'partially resistant', 'fully resistant', and 'super resistant'malaria. Trends Parasitol. 2013;29:505-15.

48. Jagannathan P, Kakuru A, Okiring J, Muhindo MK, Natureeba P, Nakalembe $M$, et al. Dihydroartemisinin-piperaquine for intermittent preventive treatment of malaria during pregnancy and risk of malaria in early childhood: a randomized controlled trial. PLoS Med. 2018;15:e1002606.
49. Sundell K, Jagannathan P, Huang L, Bigira V, Kapisi J, Kakuru MM, et al. Variable piperaquine exposure significantly impacts protective efficacy of monthly dihydroartemisinin-piperaquine for the prevention of malaria in Ugandan children. Malar J. 2015;14:368.

50. Gbedande K, Varani S, Ibitokou S, Houngbegnon P, Borgella S, Nouatin O, et al. Malaria modifies neonatal and early-life toll-like receptor cytokine responses. Infect Immun. 2013;81:2686-96.

51. Rachas A, Le Port A, Cottrell G, Guerra J, Choudat I, Bouscaillou J, et al. Placental malaria is associated with increased risk of non malaria infection during the first 18 months of life in a Beninese population. Clin Infect Dis. 2012;55:672-8.

52. Dechavanne C, Cottrell G, Garcia A, Migot-Nabias F. Placental malaria: decreased transfer of maternal antibodies directed to Plasmodium falciparum and impact on the incidence of febrile infections in infants. PLoS ONE. 2015;10:e0145464.

53. Moro L, Bardaji A, Nhampossa T, Mandomando I, Serra-Casas E, Sigauque $B$, et al. Malaria and HIV infection in Mozambican pregnant women are associated with reduced transfer of antimalarial antibodies to their newborns. J Infect Dis. 2015;211:1004-14.

54. Desai M, Gutman J, Taylor SM, Wiegand RE, Khairallah C, Kayentao K, et al. Impact of sulfadoxine-pyrimethamine resistance on effectiveness of intermittent preventive therapy for malaria in pregnancy at clearing infections and preventing low birth weight. Clin Infect Dis. 2016;62:323-33.

\section{Publisher's Note}

Springer Nature remains neutral with regard to jurisdictional claims in published maps and institutional affiliations.
Ready to submit your research? Choose BMC and benefit from:

- fast, convenient online submission

- thorough peer review by experienced researchers in your field

- rapid publication on acceptance

- support for research data, including large and complex data types

- gold Open Access which fosters wider collaboration and increased citations

- maximum visibility for your research: over 100M website views per year

At BMC, research is always in progress.

Learn more biomedcentral.com/submissions 